\title{
Outcome of Neonates Born to COVID-Positive Women at 6 Months of Age
}

\author{
Dinesh Munian, ${ }^{\mathbf{1}}$ Rituparna Das, ${ }^{\mathbf{1}}$ AviJit Hazra, ${ }^{\mathbf{2}}$ Somosri RaY ${ }^{\mathbf{1}}$ \\ From Department of ${ }^{l}$ Neonatology, Medical College and Hospital, Kolkata, West Bengal; ${ }^{2}$ Department of Pharmacology, Institute of \\ Postgraduate Medical Education \& Research (IPGME\&R) and SSKM Hospital, Kolkata, West Bengal.
}

Correspondence to: Dr Somosri Ray, Department of Neonatology, Medical College and Hospital, Kolkata 700073 , West Bengal.dr.somosri@gmail.com Received: March 13, 2021;

Initial review: April 15, 2021; Accepted: July 09, 2021.
Objective: To compare clinical and neurodevelopmental outcome at the age of 6 months for neonates born to SARS-CoV-2-positive mothers. Methods: Neonates of SARS-CoV-2 positive mothers, admitted in our hospital were assessed for growth, neurodevelopment by Amiel-Tison method, and Developmental Profile (DP3) at discharge as part of another study (July 2020). This data were retrieved and babies followed-up at the age of 6 months. Composite adverse outcome was death within 6 months post discharge or DP3 score $<70$ and hearing/visual deficit. Results: Out of 131 enrolled at discharge, $127(97 \%)$ were followed up. SARS-CoV-2 positive neonates (Group I; 19, 15\%) had more symptoms $(P=0.012)$, sepsis $(P=0.014)$, pneumonia $(P=0.029)$, longer hospital stay $(P<0.001)$ following birth compared to group II (SARS-CoV-2 negative neonates;108, 85\%). No baby in group I met definition of composite adverse outcome, while in group II it was $0.9 \%$ (1 child with DP3 $<70$ with hearing deficit) $(P=1.0)$ without any difference in hospital readmission, growth, DP3 scores, or tone abnormalities. Conclusions: There is no difference in growth, neurodevelopment, and hospital readmission in early infancy among infected and non-infected babies born to SARS-CoV-2 positive mothers.

Keywords: Corona virus, SARS-CoV-2, Neonate, Neurodevelopment.

Published online: July 23, 2021; PII:S097475591600354

$\mathrm{A}$ dverse pregnancy outcomes have been documented with two earlier pathogenic coronavirus infections - severe acute respiratory syndrome (SARS) and Middle East respiratory syndrome (MERS) [1]. However, most severe acute respiratory syndrome coronavirus 2 (SARSCoV-2) -positive neonates (50\%) were symptomatic with predominant respiratory symptoms attributed to coronavirus disease (COVID) [2,3] and required intensive care. Among symptomatic SARS-CoV-2 positive neonates, morbidities also relate to prematurity and perinatal events [4].

Information on long term outcome of neonates following COVID-19 is lacking so far. Only a handful of studies are available following SARS $[5,6]$. Hence, we planned to assess clinical and neurodevelopmental outcome in early infancy for neonates born to SARSCoV-2 positive mothers.

\section{METHODS}

After institutional ethics committee approval, information was retrieved from hospital records for the present study. Demographic details, clinical features, hospital course, and SARS-CoV-2 positively status were collected for all neonates born to SARS-CoV-2 positive mothers during May to July, 2020, as part of a previous study [unpublished data].

INDIAN PEDIATRICS
Nasopharyngeal and oropharyngeal swabs for COVID-19 real time-polymerase chain reaction (RT-PCR) were sent at 24-48 hours of life [7]. For outborns, if admitted beyond 48 hours, RT-PCR test was done at admission. The test was repeated immediately, if new symptoms appeared, even if the first test was negative; otherwise test was repeated after 5 days. For SARS-CoV-2 positive neonates, repeat test was done after 10 days and they were discharged, if negative.

After parental consent, the children were assessed in the neonatal follow-up clinic at 14 days following discharge, then at 6 weeks, 3 months and 6 months of corrected age. Weight, length and head circumference were measured using electronic weighing scale, infantometer, non-stretchable fiberglass tape, respectively and plotted on WHO growth chart [8]. The advanced or delayed development across five domain scores physical, adaptive behavior, social-emotional, cognitive and communication - and the general development score were plotted at 6 months of corrected age as per Developmental Profile 3 (DP3) manual by a single investigator [9]. Children were classified as per following scheme: Score $<70$ - delayed, 70-84 - below average, 85 114 - average, 115-130 - above average, and $>130$ - well above average.

Neurological examination was done by a single 
investigator as per Amiel-Tison method [10]. Retinopathy of prematurity (ROP) screen, if indicated, and brainstem evoked response audiometry (BERA) with age-appropriate behavioral audiometry were done at follow-up. During follow-up, parents were interviewed with pre-tested and pre-validated questionnaire containing questions on details of their baby's readmission (if any till date). The details of readmission were confirmed by checking the discharge certificates or verified from medical records if readmitted in our hospital. All babies readmitted in our hospital underwent RT-PCR for SARS-CoV-2.

Primary outcome was adverse composite outcome defined as death within 6 months post discharge or developmental delay (defined as DP3 score $<70$ ) with hearing/visual deficit. Secondary outcomes were DP3 scores, hearing, visual deficit, abnormal tone, growth $z$ scores at follow-up, hospital readmission rate, noninvasive/invasive respiratory support days during readmission.

Statistical analysis: Numerical variables were compared between groups by Student independent samples $t$ test, if normally distributed or by Mann-Whitney $U$ test, if otherwise. Fisher exact test or Pearson chi-square was employed for intergroup comparison of categorical variables. All analyses were two-tailed and statistical significance was set at $P<0.05$ for all comparisons.

\section{RESULT}

Out of 131 enrolled neonates, results of 127 (97\%) babies were analyzed (Fig. 1). All mothers were RT-PCR positive at median (IQR) of 5 [2,8] days before delivery. All symptomatic SARS-CoV-2 positive neonates $(n=10)$ had sepsis like manifestations (Table I). None had meconium aspiration syndrome, hyaline membrane disease or moderate to severe perinatal asphyxia. SARS-CoV-2 positive neonates (group I) were more symptomatic $(P=0.012)$, more commonly had sepsis $(P=0.014)$ or pneumonia $(P=0.029)$, and had longer duration of hospital stay $(P<0.001)$ compared to group II.

There was no death post-discharge. During followup, no infant in group I met definition of composite adverse outcome, while in group II one child $(0.9 \%)$ had DP3 score $<70$ with hearing deficit $(P=1.0)$. There were no differences in DP3 scores and anthropometry among the two groups (Table II). BERA was done in 2 out of 19 babies in group I, which was normal in all, while in group II, it was done in 10 babies and was normal in 9 babies $(P=1.0)$. No baby had abnormal ROP (done for only 9 babies). No babies other than one with delayed development in group II had abnormal tone.

Seven babies from group II (pneumonia 3, bronchiolitis 1 , viral upper respiratory infection and diarrhea 1 , sepsis with poor feeding and lethargy 2) and two from group I (bronchiolitis 1, diarrhea 1) were readmitted. SARS-CoV-2 RT-PCR were negative in all 9 readmitted babies. None required noninvasive or invasive mode of ventilation following readmission. There was no difference in course on readmission (Table II).

\section{DISCUSSION}

In our study, no SARS-CoV-2 positive neonate in infancy met definition of Composite adverse outcome, at 6 months while it was $0.9 \%$ in the other group.

Neonates are said to be exposed to SARS-CoV-2 if they are born to the mothers with a history of SARSCoV-2 infection diagnosed 14 days before or 28 days after

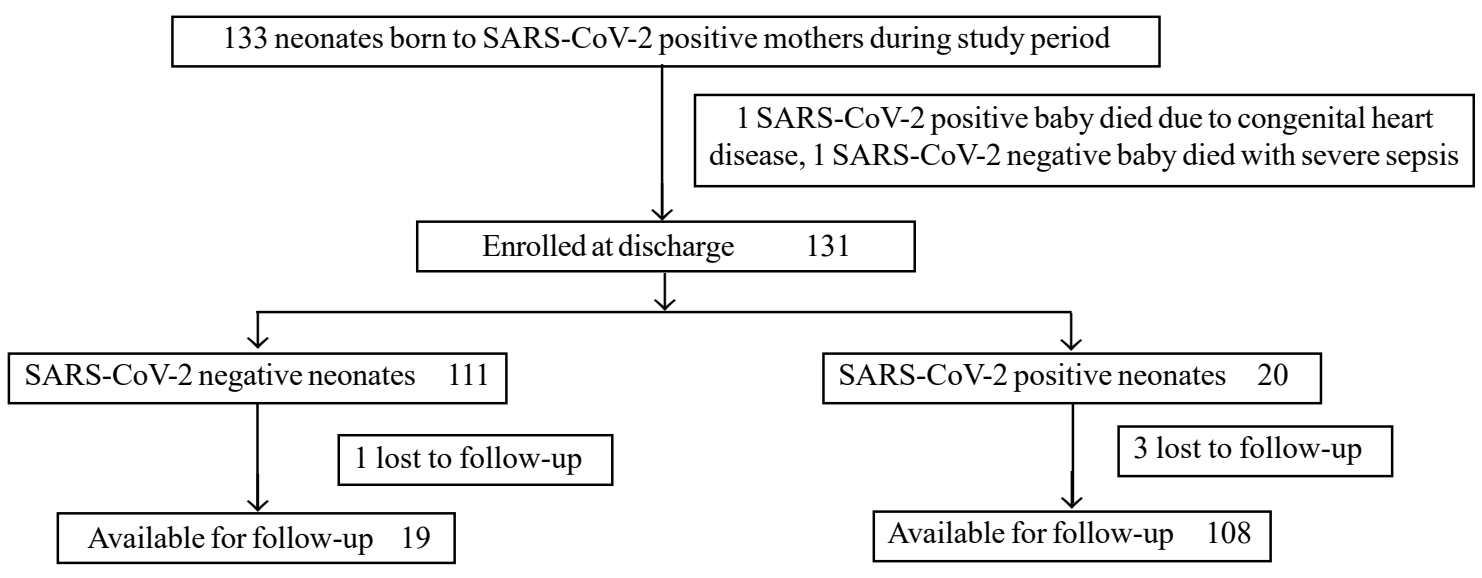

Fig. 1 Study flow chart. 
Table I Demographic and Clinical Details of Neonates Born to SARS-CoV-2 Positive Mothers $(N=127)$

\begin{tabular}{|c|c|c|}
\hline Parameters & $\begin{array}{l}S A R S-C o V-2 \\
\text { positive } \\
(n=19)\end{array}$ & $\begin{array}{r}S A R S-C o V-2 \\
\text { negative } \\
(n=108)\end{array}$ \\
\hline Gestational age $(\mathrm{wk})^{a}$ & $37(36,38)$ & $37(36,38)$ \\
\hline Birthweight $(\mathrm{g})^{a}$ & $\begin{array}{l}2765 \\
(2300,3135)\end{array}$ & $\begin{array}{r}2700 \\
(2231,3000)\end{array}$ \\
\hline Male sex & $12(63.1)$ & $62(57.4)$ \\
\hline Small for gestational age & $4(21)$ & $28(25.9)$ \\
\hline Vaginal delivery & $14(73.7)$ & $66(61.1)$ \\
\hline Age at RT-PCR sampling (h) ${ }^{a}$ & $48(38,96)$ & $48(40,79)$ \\
\hline Hospital stay after birth (d) $)^{a, b}$ & $10(8,16)$ & $6(3,7)$ \\
\hline Symptomatic babies $^{c}$ & $10(52.6)$ & $23(21.3)$ \\
\hline Respiratory distress & $7(36.8)$ & $21(19.4)$ \\
\hline Transient tachypnea of newborn & $1(5.2)$ & $6(5.5)$ \\
\hline Pneumonia $^{\mathrm{d}}$ & $4(21)$ & $5(4.6)$ \\
\hline Poor feeding/lethargy & $3(15.7)$ & $7(6.5)$ \\
\hline Vomiting & $2(10.5)$ & $7(6.5)$ \\
\hline Diarrhea & $2(10.5)$ & $3(2.7)$ \\
\hline Hypothermia & $1(5.3)$ & $2(1.8)$ \\
\hline Shock & $1(5.2)$ & $1(0.9)$ \\
\hline Seizure & $2(10.5)$ & $2(1.8)$ \\
\hline Probable sepsis ${ }^{c, e}$ & $9(47.4)$ & $19(17.6)$ \\
\hline Culture positive sepsis & $1(5.3)$ & $3(2.7)$ \\
\hline Meningitis & $1(5.3)$ & $2(1.8)$ \\
\hline Duration of antibiotics (d) ${ }^{a}$ & $6(5,14)$ & $7(5,8.5)$ \\
\hline Duration of oxygen $(\mathrm{h})^{a}$ & $48(39,84)$ & $42(24,48)$ \\
\hline
\end{tabular}

Data in no. (\%) or ${ }^{a}$ Median (IQR). RT-PCR: Real time polymerase chain reaction, TTNB: Transient tachypnea of newborn. ${ }^{b} P<0.001$, ${ }^{c} P=0.001 .{ }^{d} P=0.03$. ${ }^{e}$ Sepsis screen positive culture negative sepsis accounted for probable sepsis. SARS-CoV-2: Severe acute respiratory syndrome coronavirus 2. Positive/negative as per real time polymerase chain reaction (RT-PCR).

delivery, or if the neonate is directly exposed to close contacts with SARS-CoV-2 infection [11]. In our study, all mothers were positive in the third trimester, within 14 days of delivery. In the absence of testing amniotic fluid or cord blood [12], it was not possible to pinpoint the timing of acquisition and mode of transmission of SARS-CoV-2 in our neonates. In our study, all the symptomatic SARSCoV-2 positive neonates had sepsis like clinical presentation; it is difficult to interpret whether the clinical course was more influenced by sepsis or SARS-CoV- 2 .

Till date, no published data on long term outcome of SARS-CoV-2 recovered neonates are available with which our findings may be compared. A multicenter cohort study from 11 hospitals in Massachusetts described
Table II Follow-up Data at 6 Months for Neonates Born to SARS-CoV-2 Positive Mothers

\begin{tabular}{|c|c|c|}
\hline Parameters & $\begin{array}{l}S A R S-C o V-2 \\
\text { positive } \\
(n=19)\end{array}$ & $\begin{array}{r}S A R S-C o V-2 \\
\text { negative } \\
(n=108)\end{array}$ \\
\hline Weight $(g)^{a}$ & $6850(893)$ & $6820(754)$ \\
\hline$<-3 z$-score & 2 & 10 \\
\hline-3 to $-2 \mathrm{z}$ score & 3 & 9 \\
\hline-2 to $0 \mathrm{z}$ score & 10 & 75 \\
\hline 0 to $+2 \mathrm{z}$ score & 3 & 12 \\
\hline+2 to $+3 \mathrm{z}$ score & 1 & 2 \\
\hline Length $(\mathrm{cm})^{a}$ & $64.8(3.1)$ & $64.9(2.6)$ \\
\hline$<-3 z$ score & 2 & 10 \\
\hline-3 to -2 z score & 4 & 9 \\
\hline-2 to $0 \mathrm{z}$ score & 9 & 75 \\
\hline 0 to $+2 \mathrm{z}$ score & 3 & 11 \\
\hline+2 to +3 score & 1 & 3 \\
\hline Head circumference $(\mathrm{cm})^{a}$ & $41.6(1.6)$ & $41.5(1.4)$ \\
\hline$<-3$ z score & 1 & 4 \\
\hline-3 to $-2 \mathrm{z}$ score & 3 & 11 \\
\hline-2 to -1 z score & 3 & 52 \\
\hline-1 to $0 \mathrm{z}$ score & 8 & 27 \\
\hline 0 to $+1 \mathrm{z}$ score & 4 & 14 \\
\hline \multicolumn{3}{|l|}{ Development assessment } \\
\hline General developmental score ${ }^{c}$ & $87.4(12.3)$ & $90.6(10.1)$ \\
\hline \multicolumn{3}{|l|}{ Developmental category $^{c}$} \\
\hline Below average & $8(42.1)$ & $28(25.9)$ \\
\hline Delay & $1(5.2)$ & $1(0.9)$ \\
\hline \multicolumn{3}{|l|}{ Readmission related } \\
\hline Babies readmitted $^{c}$ & $2(10)$ & $7(6.5)$ \\
\hline Age at readmission $(\mathrm{d})^{a}$ & $105(21)$ & $68(45)$ \\
\hline Duration of antibiotic $(\mathrm{d})^{a}$ & $3(0)$ & $5.3(1.5)$ \\
\hline Duration of oxygen (d) $(n=7)^{b}$ & $3(0,0)$ & $4(3,4)$ \\
\hline
\end{tabular}

DP3:Developmental profile 3, BERA: Brainstem evoked response audiometry, ROP: Retinopathy of prematurity. ${ }^{a}$ Mean (SD), ${ }^{b}$ Median (IQR), ${ }^{c} n(\%)$. All $P>0.05$.

short term follow up of 151 newborns born to SARSCoV-2 positive mothers, till 30 days of hospital discharge although growth, neurodevelopment were not incorporated [13]. In this study, four babies were rehospitalised, due to laryngomalacia, hyperbilirubi-nemia, ventricular arrhythmia and blood culture positive sepsis, respectively, none directly associated with SARS-CoV-2 infection [13]. Another follow up study from New York showed follow up till day 25 in 23 out of 101 babies born to SARS-CoV-2 positive mothers [14], 4 having readmissions, 3 for fever and 2 for hyperbilirubinemia, 


\section{WHAT THIS STUDY ADD?}

- There is no difference in growth and neurodevelopment, and rate of hospital readmission in early infancy among SARS-CoV-2 positive and negative neonates born to mothers with perinatal SARS-CoV-2 infection.

none having evidence of SARS-CoV-2 reinfection. Several follow-up studies since the previously known pathogenic corona viral infection outbreak - SARS (20022003 ) are there. The outcomes in children up to 6 months after SARS disease onset, in terms of exercise tolerance, pulmonary function and psychologic status, have been favorable [5,6]. All children post-SARS were found to remain clinically asymptomatic till next 6 month; although, with mild obstructive or restrictive defect on pulmonary function study in $10 \%$ of them [15]. Pulmonary function test could be done in our cohort later in life.

The limitations of this study was that only illness severe enough to require hospital admission was considered, which may have left out morbidities like fever, cough and cold controlled with over the counter medicines. Moreover, the person assessing the neurodevelopment was not blinded to the group-assignment. Despite these shortcomings, we may reasonably conclude that there are no differences in growth, neurodevelopment, and hospital readmission in early infancy between SARS-CoV-2 positive and negative neonates born to SARS-CoV-2 positive mothers.

Ethics clearance: Institutional Ethics Committee of Medical College Kolkata; No. MC/KOL/IEC/NON-SPON/1046/02/ 2021, dated February 20, 2021.

Contributors: SR: substantial contribution in acquisition, analysis of data, drafting the work; DM: substantial contribution in design of the work, interpreting the data, revising it critically for important intellectual content; RD: substantial contribution in acquisition of data, interpretation of results and critical revision of the work; AH: substantial contribution in conception, analysis of data, critical revision of the work. All authors approved the final version to be published.

Funding: None; Competing interest: None stated.

\section{REFERENCES}

1. Schwartz DA, Graham AL. Potential maternal and infant outcomes from Coronavirus 2019-nCoV (SARS-CoV-2) infecting pregnant women: Lessons from SARS, MERS, and other human coronavirus infections. Viruses. 2020;12:194.

2. Dhir SK, Kumar J, Meena J, Kumar P. Clinical features and outcome of SARS-CoV-2 infection in neonates: A systematic review. J Trop Pediatr. 2020;28:fmaa059.

3. Raschetti R, Vivanti AJ, Vauloup-Fellous C, et al. Synthesis and systematic review of reported neonatal SARS-CoV-2 infections. Nat Commun. 2020;11:5164.

4. National Neonatology Forum (NNF) COVID-19 Registry Group. Outcomes of neonates born to mothers with coronavirus disease 2019 (COVID-19) - National Neonatology Forum (NNF) India COVID-19 Registry. Indian Pediatr. 2021:58:525-31.

5. Leung CW, Kwan YW, Ko PW, et al. Severe acute respiratory syndrome among children. Pediatrics. 2004; 113:e535-43.

6. Li AM, Chan CH, Chan DF. Long-term sequelae of SARS in children. Paediatr Respir Rev. 2004;5:296-99.

7. Chawla D, Chirla D, Dalwai S, et al. Perinatal-Neonatal Management of COVID-19 infection - Guidelines of the Federation of Obstetric and Gynaecological Societies of India (FOGSI), National Neonatology Forum of India (NNF), and Indian Academy of Pediatrics (IAP). Indian Pediatr. 2020;57:536-48.

8. World Health Organization. New WHO growth charts. Accessed on May 26, 2021. Available from: https:// www.who.int/nutgrowthdb/software/en/

9. Alpern GD. Developmental profile 3 (DP-3). Western Psychological Services; 2007.

10. Amiel-Tison C. Neurological evaluation of the maturity of newborn infants. Arch Dis Child. 1968;43:89-93.

11. Wang L, Shi Y, Xiao T, et al. Chinese expert consensus on the perinatal and neonatal management for the prevention and control of the 2019 novel coronavirus infection (First edition). Ann Transl Med. 2020;8:47-57.

12. Goh XL, Low YF, Ng CH, Amin Z, Ng YPM. Incidence of SARS-CoV-2 vertical transmission: A meta-analysis. Arch Dis Child Fetal Neonatal Ed. 2021;106:112-3.

13. Angelidou A, Sullivan K, Melvin PR et al. Association of Maternal Perinatal SARS-CoV-2 infection with neonatal outcomes during the COVID-19 pandemic in Massachusetts. JAMA Netw Open. 2021;4:e217523.

14. Dumitriu D, Emeruwa UN, Hanft E et al. Outcomes of neonates born to mothers with severe acute respiratory syndrome coronavirus 2 infection at a large medical center in New York City. JAMA Pediatr. 2021;175:157-67.

15. Li AM, So HK, Chu W et al. Radiological and pulmonary function outcome of children with SARS. Pediatr Pulmonol. 2004;38:427-33. 